Ann. Génét. Sél. anim., I970, 2 (3), 269-272.

\title{
PROPORTION DES SEXES CHEZ LES VOLAILLES : VARIATION DANS LA DESCENDANCE DU MÊME PÈRE SUIVANT LA DATE D'ÉCLOSION
}

\author{
P. MERAT \\ Station centrale de Génétique animale, \\ Centre national de Recherches zootechniques, 78-Jouy-en-Josas \\ Institut national de la Recherche agronomique
}

\section{RÉSUMÉ}

Une variation de la proportion des sexes, apparemment non aléatoire $(P<0,01)$, a été trouvée entre couvées successives issues d'un même père, dans une population étudiée de 1955 à 1963. Elle n'est pas « synchronisée " pour tous les péres à la même date. Il semble douteux que cette variation s'explique entièrement par des fluctuations dans le temps, pour chaque père, d'une mortalité embryonnaire différentielle entre sexes.

Cette hétérogénéité entre dates d'éclosion intra-pères parait présente aussi dans d'autres populations.

D'autre part, l'hétérogénéité hautement significative trouvée précédemment entre pères est confirmée. Elle ne peut être due seulement à la variation " entre dates, intra-pères ".

Dans une analyse portant sur environ 170 ooo poussins pedigree, nous avions mis en évidence une hétérogénéité hautement significative de la proportion des sexes (" sex ratio ") à 8 semaines d'âge et à l'éclosion, entre souches et entre familles (MÉRAr, I963). Une interprétation par une mortalité différentielle des deux sexes variable suivant les familles était très peu vraisemblable, les familles à excès significatif d'un sexe ne différant pas des autres pour la mortalité embryonnaire ou juvénile. Il paraît donc s'agir d'une hétérogénéité relative à la proportion des sexes à la fertilisation (" sex ratio primaire "). Cette conclusion est renforcée par l'étude de quelques familles particulières à proportion très anormale et à faible nombre de zygotes de sexe non identifié (MÉRAT, r969).

Les résultats présents concernent une variation apparemment non aléatoire de la " sex ratio " entre semaines d'éclosion successives, à l'intérieur des descendances de même père. 
Comme nous l'avons déjà indiqué dans les publications citées, les données utilisées proviennent de plusieurs souches et races élevées respectivement à la Station avicole de Jouy-en-Josas, à la Station du Magneraud et dans plusieurs élevages de sélection. Dans tous les cas, chaque père était accouplé à un nombre variable de femelles, mais chaque poule à un seul coq. Le sexe était déterminé à 8 semaines et vérifié à Io, ainsi que sur les embryons morts après $\mathrm{I} 8$ jours d'incubation depuis I959 à Jouy. Aucune élimination volontaire n'avait lieu avant l'âge de ro semaines.

Nous étudierons essentiellement ici la population "Jouy ", issue d'un croisement initial et reproduite ensuite sans apport extérieur de I955 à I963.

Le tableau I donne, pour l'ensemble des années, l'analyse de 1'hétérogénéité par décomposition $\mathrm{du} \chi^{2}$ en trois éléments : écart global à la proportion $\mathrm{I} / \mathrm{I}$, hétérogénéité entre descendances de pères différents, enfin hétérogénéité entre dates d'éclosion intra-pères.

\section{TABLEAU I}

Analyse de l'hétérogénéité de la "sex ratio "par pères et dates d'éclosion

\begin{tabular}{|c|c|c|c|}
\hline Source de variation & $\begin{array}{c}\text { Degrés } \\
\text { de liberté }\end{array}$ & $x^{2}$ & Signification \\
\hline $\begin{array}{l}\text { Écart global } \ldots \ldots \ldots \ldots \ldots \\
\text { Hétérogénéité entre pères } \ldots \ldots \\
\text { Hétérogénéité entre dates d'éclo- } \\
\text { sion intra-pères } \ldots \ldots \ldots \ldots \ldots\end{array}$ & $\begin{array}{r}I \\
450 \\
2312\end{array}$ & $\begin{array}{r}0,16 \\
553,62 \\
2503,87\end{array}$ & $\begin{array}{l}\text { N.S. } \\
\mathbf{P}<\text { o,OoI } \\
\mathbf{P}<\text { o,oI }\end{array}$ \\
\hline
\end{tabular}

L'hétérogénéité très hautement significative entre pères a déjà été notée (MÉRAT, I963). De plus, un élément nouveau apparaît ici, 1'hétérogénéité, significative au seuil I $p$. Ioo, entre dates d'éclosion intra-pères.

Cette dernière ne paraît pas refléter une variation d'ensemble de la proportion des sexes d'une date d'éclosion à l'autre, car le $\chi^{2}$ " entre dates, intra-années ", tous les pères d'une année étant groupés, est égal à I 85,43 pour I 78 degrés de liberté (non significatif). La variation apparemment non aléatoire entre dates d'éclosion serait donc propre à chaque descendance, sans "synchronisme " entre les différents pères $\left({ }^{1}\right)$.

Pour apprécier correctement la signification du $\chi^{2}$ " intra-pères ", il faut toutefois tenir compte de l'hétérogénéité importante qui existe entre reproducteurs mâles (HowARD et al., I955). Or, l'examen des variations de la proportion totale suivant le père montre que, si l'on corrigeait les $\chi^{2}$ " intra-pères " d'après cette proportion globale $\left({ }^{2}\right)$, la réduction de ces $\chi^{2}$ ne pourrait être que faible, vraisem-

(1) Il ne semble pas non plus que la variation entre dates soit plus grande pour des pères particuliers, notamment ceux présentant globalement la proportion des sexes la plus déviée. (2) Ce qui revient à multiplier chaque $x^{2}$ d'hétérogénéité intra-père par $\frac{I}{4 \hat{p} \hat{q}}, \hat{p}$ étant la proportion
observée relative au père en question. 
blablement inférieure à I p. Ioo dans l'ensemble, ce qui ne modifierait pas la signification du résultat.

Cette hétérogénéité entre périodes successives pour le même père n'est probablement pas un phénomène particulier à la population "Jouy ", car, sur l'ensemble des autres populations étudiées, le $\chi^{2}$ correspondant est égal à 3826,89 pour 3678 degrés de liberté $(\mathrm{P} \simeq 0,05)$.

On peut se demander si la variation observée est attribuable à des changements de la mortalité différentielle des deux sexes d'une date à l'autre pour la descendance d'un père donné. Pour tenter de le vérifier, nous avons d'abord comparé la proportion des sexes dans les deux groupes suivants : le premier contenant, pour chaque père, le lot (descendance éclose le même jour) ayant eu le meilleur taux d'éclosion (poussins nés /œufs incubés), le second constitué en prenant, pour chaque père, le lot ayant eu la plus mauvaise éclosion. Sur ro 463 enfants représentant le premier groupe, la proportion de mâles est de 49,92 p. Ioo, contre 50,53 p. IOo pour 7769 enfants du deuxième groupe. La différence n'est pas significative $\left(\chi^{2}=0,63\right.$ pour I d.1.).

Inversement, nous avons choisi, pour chaque père, le " lot " de naissance dans lequel l'excès le plus caractérisé de mâles était trouvé (d'après la valeur $\mathrm{du} \chi^{2}$ de conformité à la porportion I / I). Sur l'ensemble de ces " lots ", le taux global d'éclosion est comparé à celui obtenu en prenant, pour chaque père, le " lot " ayant donné l'excès le plus net de femelles (d'après le $\chi^{2}$ ). Ces taux d'éclosion sont respectivement 74,37 p. IOO sur II 420 œufs incubés dans le premier cas et 75,50 p. roo sur I I 554 œufs incubés dans le second. Leur différence, quoique faible, s'approche du seuil 5 p. Ioo de signification $\left(\chi^{2}=3,89\right)$.

Cependant, en totalisant également, pour les mêmes pères, l'ensemble des lots à " sex ratio " la moins extrême, non inclus dans les deux groupes précédents, on obtient un taux d'éclosion global de 75, I 5 p. roo sur $5635^{8}$ poussins, voisin des deux précédents, et l'on vérifie qu'entre l'ensemble de ces trois groupes, il n'existe pas de différence significative $\left(\chi^{2}=4\right.$, I I pour 2 degrés de liberté, $\left.\mathrm{P}>0, \mathrm{IO}\right)$.

D'ailleurs, cette simple comparaison ne tient pas compte des variations non aléatoires importantes de la mortalité embryonnaire qui existent entre dates d'éclosion successives, et, de ce fait, sa signification est quelque peu surestimée.

De toute façon, la différence moyenne d'éclosion entre les "lots " à " sexratio " extrême pour le même père, si elle était réelle, serait minime : I p. Ioo environ. On peut douter qu'elle suffise à rendre compte de la variation observée du "sex ratio " à la naissance.

Quoiqu'on ne puisse le prouver de façon décisive avec nos données, il n'est donc pas impossible qu'il existe, pour la descendance d'un même coq, une légère variation, dans le temps, de la proportion des mâles et des femelles, non due à une mortalité embryonnaire, c'est-à-dire affectant la " sex-ratio primaire ".

On peut remarquer enfin que l'hétérogénéité considérable entre descendances globales de mâles différents (MÉRAT, r963) ne peut s'expliquer seulement par cette variation " entre lots intra-pères " comme le suggère le rapport des $\chi^{2}$ correspondant à ces deux sources de variation : $\mathrm{F}=\mathrm{I}, \mathrm{I} 37$ pour 450 et $23 \mathrm{I} 2$ d.1., d'où 
$\mathrm{P} \simeq 0,05$ d'après les moments de la loi de $z=\frac{\mathrm{r}}{2} \mathrm{~L}(\mathrm{~F})$ pour des nombres de degrés de liberté élevés (KENDali, et StuART, I963).

Ręu pour publication en mars 1970.

\section{SUMMARY}

SEX RATIO IN THE FOWL : VARIATIONS BETWEEN HATCHES WITHIN SIRE FAMILIES

An apparently non-random variation $(P<.01)$ of the sex-ratio was found between successive hatches within sires in a strain studied from 1955 to 1963 . It does not correspond to a " synchronized "variation for all sires at the same date. It seems doubtful that this variation can be explained entirely by time fluctuations, within each sire, of a differential embryonic mortality between sexes.

This heterogeneity between hatches within sires seems to be present also in other populations.

on the other hand, the highly significant heterogeneity found previously between sire families is confirmed, and it cannot be explained only by the "between hatches withinsires" variation.

\section{RÉFÉRENCES BIBLIOGRAPHIQUES}

Howard A., Mc I Aaren A., Michie D., Sander G., I955. Genetic and environmental influences on the secondary sex ratio in mice. $J$. Genet., 53, 200-2r4.

Kendall M. G., Stuart A., 1963. The advanced theory of statistics. Vol. I, Griffin and Co., I,ondon, $2^{\mathrm{e}}$ éd.

Mérat P., r963. Proportion des sexes (sex ratio) chez les volailles. II. Différences entre souches et entre familles. Ann. Zootech., 12, ro5-123.

MÉrat P., r969. Proportion anormale des sexes chez les volailles : Examen de familles particulières. Ann. Génét. Sélect. anim., 1, I47-I 50 